\title{
A change of culture: reducing blood culture contamination rates in an Emergency Department
}

\author{
James Bentley, Shobhan Thakore, L Muir, Alastair Baird, Jennifer Lee
}

To cite: Bentley J, Thakore S, Muir L, et al. A change of culture: reducing blood culture contamination rates in an Emergency Department. BMJ Quality Improvement Reports 2016;5:u206760. w2754. doi:10.1136/ bmjquality.u206760.w2754

Received 23 February 2016 Revised 26 April 2016

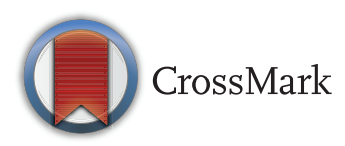

NHS Tayside, Scotland

Correspondence to James Bentley jamesbentley@nhs.net

\section{ABSTRACT}

Blood cultures are an important investigation to help tailor effective management for patients with severe sepsis. Frequent contaminated samples increase laboratory workload and can delay or cause incorrect changes to patient management. This can prolong patient hospitalisation, increase the risk of harm and increase cost to health boards. Current guidelines advocate a contamination rate of 2-3\%. From January 2013 to November 2014 inclusive, the contamination rate was $4.74 \%$ in our Emergency Department, responsible for initial management and investigation of over 40 cases of sepsis per month. A Quality Improvement team was created to try to reduce contamination rates to the recommended target.

An initial baseline survey of local staff showed good understanding of when to obtain a blood culture but there was variability in the methods and equipment used. A project was then conducted which focused on rationalising and standardising equipment and technique for blood culture sampling along with staff education to support this change. A simple department target of 30 days free from a contaminated blood culture was created which, if achieved, would ensure a contamination rate of less than $3 \%$. This was supported by ongoing surveillance of contamination rates and investigation of contaminated sample cases. We were able to then identify high risk patients and factors which increased the chance of blood culture contamination. This allowed us to formulate solutions to help reduce the risks of contamination. Department achievements and learning points to help prevent further contamination were fed back positively to all staff. This project operated for 12-months and successfully reduced local contamination rates to $2.0 \%$.

\section{PROBLEM}

Blood culture (BC) contamination rates locally have been consistently greater than the international recommended rate of $3 \% .^{1}$ Ninewells Emergency Department (ED) is part of a university affiliated teaching hospital in Dundee, Scotland and sees around 50,000 undifferentiated cases per annum with up to 40 patients admitted with sepsis per month. From 1st January 2013 to 30th November 2014, 1477 BC samples were taken with 70 samples growing non-clinically significant organisms giving a mean false positive (contamination) rate of $4.74 \%$.

\section{BACKGROUND}

BCs are an important laboratory investigation when managing patients with sepsis. ${ }^{2}$ Subsequent information identifying: cause of bacteraemia; organism sensitivities; exotoxins; multidrug resistance or organisms which need appropriate infection control can all be invaluable in tailoring the most effective and specific management for patients. ${ }^{3}{ }^{4}$ Initial treatment with empirical broad-spectrum antibiotics is recommended with a view to focused treatment, guided by BC results, as soon as possible. ${ }^{3}$ Inappropriate antibiotics use increases the development of multi-resistant strains of organisms, antibiotic associated colitis and the potential for adverse drug reaction..$^{5-8}$ Thus, to focus treatment early and reduce the occurrence of these issues clinical decisions are often made on early interim results.

Organisms, existing as skin flora, can appear in BC samples and be of no clinical significance. These are termed 'contaminated' or false positive samples. ${ }^{5} 9{ }^{10}$. In certain clinical situations, however, these usually insignificant colonising organisms can be the cause of significant bacteraemias. ${ }^{11}$ Although patients with clinical signs of sepsis have an increased chance of a positive culture, no clinical measurement has proved useful in distinguishing between the likelihood of contamination versus true positive cultures. ${ }^{12-14}$ This poses challenges for laboratories in deciding which samples to investigate fully and for clinicians in how they alter patient management based on initial results. ${ }^{915} 16$ Currently, all positive BCs receive a complete laboratory work-up taking in excess of 48 hours to identify organisms. ${ }^{9}$ Further investigation may then be needed to conclude whether this organism is the likely causative organism. If early clinical decisions 
are based on contaminated BCs patients may be subjected to needless risk as well as health boards subjected to increased, unnecessary costs. ${ }^{15} 16$

There have been a number of suggestions as to why BC contaminations occur along with methods for reducing contamination investigated. ${ }^{17-20}$ Recent quality improvement (QI) projects have reduced contamination rates with the introduction of sterile gloving during venepuncture. $^{20} 21$ Evidence, however, suggests most contaminants are from patient skin flora and up to $20 \%$ of skin flora may still be cultured on harvesting samples using sterile surgical techniques. ${ }^{17}$ This suggest the extra time required and equipment costs may not have any benefit over meticulous 'no-touch' peripheral venepuncture method recommended by UK Department of Health. Thus, a no-touch technique is advocated by NHS Tayside using a closed system with winged safety needle to also follow the local Sharps Injury Prevention Strategy. ${ }^{1}{ }^{22}$ A number of papers demonstrated higher contamination rates in cultures drawn from central venous catheters compared with peripheral venepuncture. ${ }^{17} 2324$ This has been assumed to be the case for samples drawn from peripheral venous catheters, a method often used in the acute setting when managing technically challenging patients. This assumption has not been supported by a recent ED based study. ${ }^{25}$ Venous cannulation is a more complex process than venepuncture and will allow more opportunities for and increased chance of contaminating samples. NHS Tayside, therefore, discourage this method but accept it when venous catheter sampling, from a freshly sited cannula, is the only route available.

Achieving proportionately lower contamination rates to overall positive results increases the reliability of a positive result being a true pathogen; thus an international target has been set at 2-3\%. ${ }^{1} 7{ }^{17}$ EDs often provide the initial management and investigations for septic patients admitted to hospital. EDs' positive BC rates have been shown to range from $3.4 \%-7.9 \%$ with contamination rates $0.6-7.9 \% .{ }^{26}{ }^{27}$ Faced with these contamination rates clinicians cannot be sufficiently confident in initial positive BC results to alter patient management. This can delay early targeted treatment and be detrimental to patient care.

\section{BASELINE MEASUREMENT}

In order to address poor contamination rates a $\mathrm{BC}$ Contamination QI group was created. This group included doctors from Specialty Trainee (ST) Year 1 to Consultant level which reflected the clinical staff responsible for collecting blood samples in the department. BC are reported as 'query significant contaminant' on Clinisys LabCentre (CliniSys Solutions Ltd) when certain bacteria which are known to cause frequent contaminations are isolated in a BC and do not correlate with clinical history as pathogenic. ${ }^{718}$ Culture request data and contamination rates were extracted from the Labcentre system for analysis. On average 64 samples were requested per month with a mean monthly contamination rate of $4.74 \%$. Previous, unpublished, audit showed that staff were good at identifying the need for $\mathrm{BC}$ and few inappropriate samples were taken.

A spot survey of practice was conducted from the 24th to 30th November 2014. A convenience sample of 11 doctors had their practice observed by a member of the QI team. Junior doctors were deliberately sampled in greater numbers than senior staff to reflect the ratio in normal practice (junior doctors are most commonly the first assessor and expected to perform initial investigations in the majority of patients). The survey was of standard format (Data Collection Supplement), focused on access to and the availability of required equipment and the technical process of sampling a BC. There was also an opportunity to offer suggestions on how culture sampling could be improved.

The survey demonstrated a universal understanding of equipment location and necessity of culture sampling prior to antibiotic administration. In general, operators displayed the correct preparation for sampling in particular not re-palpating the vein prior to venepuncture after cleaning the site. Variability in venepuncture method was shown along with culture bottle inoculation. This generally showed senior doctors adopting the more traditional needle and syringe method $(4,36 \%)$, and junior doctors opting for the closed method $(7,64 \%)$. Most reported little deviation from their preferred method with any deviation being a deliberate action in patients with poor peripheral veins. There was a high reported rate of using cannulae as an acceptable phlebotomy method. Also, working under the 'Sepsis Six' one hour time restraint appeared to impart a perceived pressure to obtain samples hastily. ${ }^{28}$ (see Data Collection supplementary file)

\section{DESIGN}

The following aim was created in order to address the problem: by December 2015 Ninewells ED's annual BC contamination rate is to be below $3 \%$.

An ambitious, realistic and simple, target was needed in order to reduce contamination rates and focus the efforts of the department which could provide immediate feedback to stakeholders. Just over 60 BC samples per month equates to around two samples per day. This meant that a contaminated sample in every 16-17 days (32-34 samples) would give a contamination rate of 2.9$3.1 \%$. A target for 30 days without a contaminant was set. This was felt to be a realistic and obtainable target that, if achieved, would give a contamination rate of around $1.6 \%$. This also allowed a buffer for any difficulties encountered so that if the target was not achieved there would still be a reasonable chance of a contamination rate of less than $3 \%$.

The QI team comprised of an ST1, ST3, ST4 (Project Lead) and Consultant from the ED and a Data Co-ordinator from the Infection Control Team. 


\section{STRATEGY}

A series of Plan, Do, Study, Act (PDSA) cycles were conducted in order to improve the contamination rate. These have been grouped into the following themes: Standards of Practice (PDSA 1-2), Engagement and Education (PDSA 3-6), Feedback and Learning (PDSA 7-9)

PDSA 1: Initial meetings were held with senior clinicians from the Microbiology Department to discuss acceptable methods of BC sampling in the ED. Conclusions closely reflected the information detailed previously of preferred methods. It was decided not to introduce the use of sterile gloving as this would increase costs in time and resources and that reinforcing good technique may be adequate at reducing contaminations. Although high-quality technique was important there was a greater clinical importance in obtaining an appropriate BC. A 'traffic-light' coding system was created for BC sampling technique:

closed system method - green;

needle and syringe method - amber;

via-cannula method - red.

Microbiology were happy to share $\mathrm{BC}$ request data and contamination rates. Details of the date of last contaminated sample, the number of requests and the number of days since this sample were also supplied daily (Monday to Friday). They also agreed to supply patient details for any samples that were considered to be contaminated.

PDSA 2: Each clinical area of the ED (Minors, Majors and Resuscitation) has a venepuncture trolley which holds a variety of equipment that may be useful for peripheral venous access and phlebotomy. These trolleys were reviewed and stocked to ensure there was a single BC shelf with the specific equipment required for NHS Tayside's primary, 'green' method for BC sampling. This included:

aerobic and anaerobic BC bottles (Biomérieux BacT/ ALERT® Culture Media);

$70 \%$ isoproypol alcohol swabs (Mölnlycke Health Care Sterets $\left.{ }^{\circledR}\right)$;

$2 \%$ chlorhexadine in $70 \%$ isoproypol alcohol swabs (Blue Clinell@ skin wipes);

winged needle Safety-Lok ${ }^{\mathrm{TM}}$ blood collection sets (BD Vacutainer $\left.{ }^{\circledR}\right)$;

BC adapter caps and inserts (Biomérieux).

To the trolleys was attached a 'check list' (Appendix 1, Supplementary Material) with clear simple instructions, following the traffic light system, for the permitted methods for sample collection. The rest of the trolley was stocked with the other equipment required for cannulation and other laboratory blood sampling which provided the equipment for the 'amber' and 'red' methods for BC sampling. This structure made it easier for doctors to perform the correct method but allowed deviation when unavoidable.

PDSA 3: In order to engage stakeholders drop-in educational sessions were held during the initial data collection phase. The focus was on the rationale behind achieving low contamination rates and how this improves patient care. This was directed at the senior, permanent staff as they were seen to be in an ideal position to direct and encourage the rotating junior staff in correct technique.

PDSA 4: An identified difficulty in instigating an improvement in technique was the perpetual change of junior staff (Foundation Year (FY) Doctors every four months and junior ST every six months). For these doctors a two-day mandatory induction programme into local policies and protocols is delivered at the start of their post. Within this programme a talk was introduced about the department BC policy. This talk focused on: the problems created by contaminated $\mathrm{BC}$, detailing the potential risks and detrimental influence on subsequent patient care; background information on how cultures are processed, thus highlighting the increased work and cost created by a contaminated BC; the methods appropriate for BC sampling, linking them to the traffic light concept; and introduction to the 'check list' available on venepuncture trolleys.

PDSA 5: Nurses were identified as being in an ideal position to assist doctors when taking BCs as they work closely when managing patients for whom BCs are required. This meant interventions to engage nurses with the principles of the project were needed. To facilitate this, discussion took place with the senior nursing team who supported enrolling the nurses in joint responsibility for reducing $\mathrm{BC}$ contamination rates. This led to providing further drop-in education sessions for the nursing staff with emphasis on the relationship between good BC sampling practice and the Sepsis Six management bundle, which is practiced locally and fully supported by the nursing staff. ${ }^{28}$ Nurses were also included in any further correspondence with regard to contamination case investigation, department updates and dissemination of learning points.

PDSA 6: During the project it was important to maintain staff engagement and identify any patient or practice factors which could lead to contaminations. These could then be addressed so as to help reduce the $\mathrm{BC}$ rate. When a contaminated sample was identified by the Microbiology Department, the patient's ED card was reviewed and information gathered, including: age, presentation time, initial observations, SIRS criteria present, primary doctor involved in case and admission diagnosis. An email was then sent to the primary doctor involved, senior supervising doctor for the patient, the main nurse involved (from March 2014) and the project team which opened a discussion about the case. The following standard questions were also asked:

What was the indication for blood culture?

Do you recall what method you used?

a) Closed system

b) Syringe and needle

c) Via cannula

d) Do not recall 
Were there particular difficulties in collecting the sample?

a) Difficult access

b) Agitated patient

c) Other - please specify

Did you use an assistant to help you take the culture samples?

Will you do anything differently the next time you take a blood culture?

Do you have any suggestions to reduce the rate of contamination e.g. equipment, education etc.?

This stimulated a dialogue, in a non-judgemental fashion to identify recurrent technical and non-technical patient, operator and equipment factors which could be addressed to reduce the number of future contaminated samples.

PDSA: 7: Email updates were sent to all ED staff when certain events occurred:

immediately before a change of junior doctors;

when new staff joined the department;

after a BC had been found to be contaminated;

every 30 days passed contaminant free;

after special cause variation shift;

alongside staff-base feedback display updates.

In these emails the staff were frequently thanked and congratulated on their assistance and achievement. The learning points obtained from case discussions were summarised and included in these emails with repetition of the important aspects but without any identifiable information surrounding the patients or clinicians involved. This was to reinforce a no-blame environment to allow regular open discussion. These emails were designed to use positive language to highlight the salient points and encourage good practice. The update also confirmed the number of days achieved without a contaminant and this information was related back to a common shared goal of improving overall mean contamination rates and subsequently patient care.

PDSA 8: A dedicated QI display area was created in the central staff area of the ED display. This showed the daily updated 'Days since last contaminant' target poster (Appendix 2, Supplementary Material) alongside monthly charts displaying department monthly BC request and contamination rates with the department's overall mean contamination and target contamination rate. On this chart the dates of the various project events were recorded. Another chart showed the number of contaminant free days that had occurred between previous contaminated samples (Figure 1, Results Supplement).

PDSA 9: This feedback display was coupled with an evolving educational display which was updated every two months (Appendix 2, Supplementary Material). This started by displaying the original information covered in the drop-in educational sessions and junior doctor induction programme. As the project developed we identified a number of learning points which could increase the likelihood of contaminating a BC and replaced the initial display with these themes and advice on how to reduce chances of contamination. The display initially contained a lot of information which was progressively simplified and focused. The strategy was to have a simple display, with visual changes so as to catch staff attention, which would trigger memories of previously conveyed learning points.

\section{Days Between Contaminated Blood Cultures Taken in A\&E, Ninewells Hospital, \\ October 2014 - November 2015}

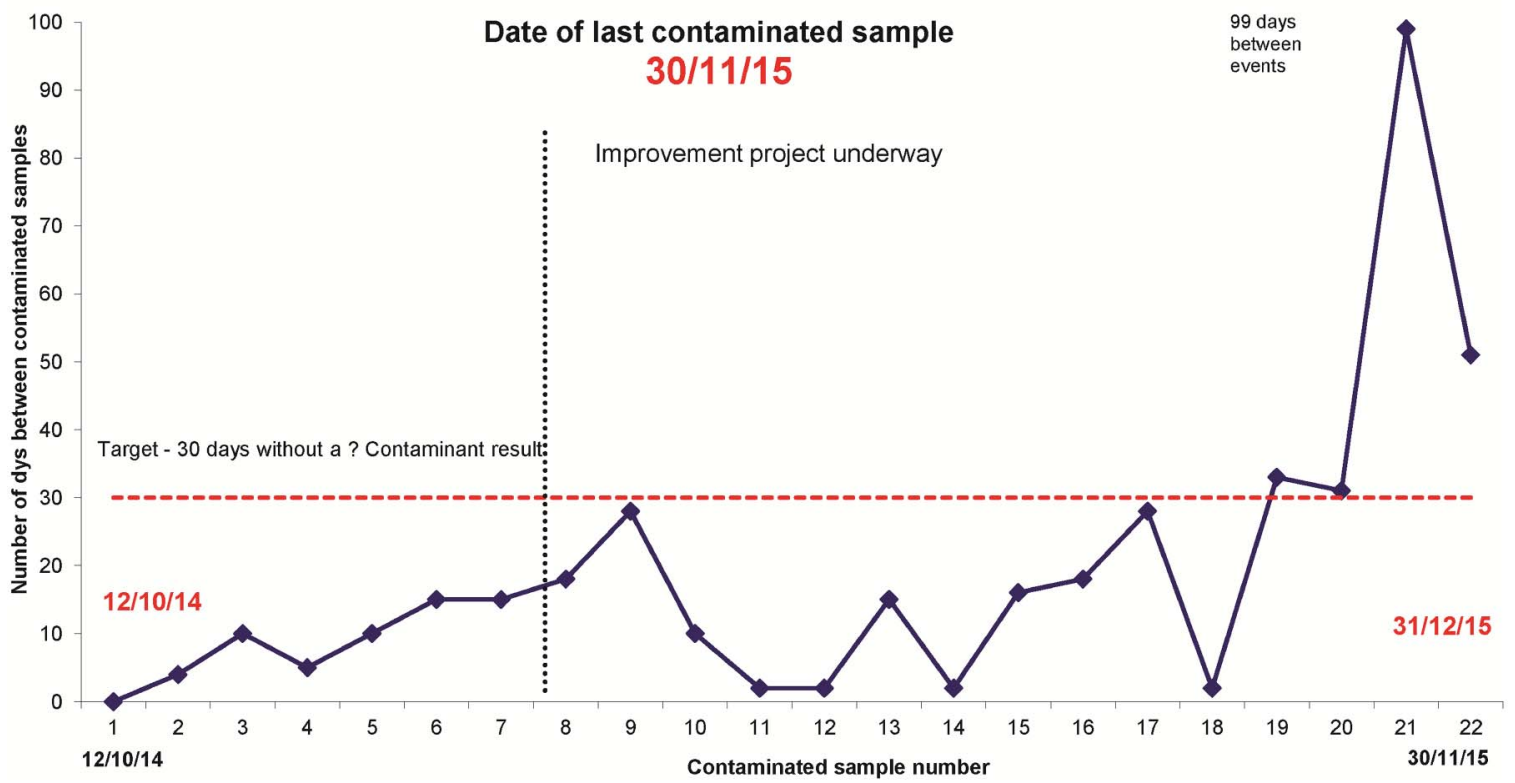

Figure 1 Chart presenting number of days between contaminated BCs for department display 


\section{POST-MEASUREMENT}

Data were collected continuously from 1st December 2014 to 30th November 2015 and were used to guide PDSA cycles 5-9. The study period saw four changeover periods with one being all Junior Doctors, one being ST only and the other two being FY only. A follow-up spot survey was performed one month after the initial survey and initiation of the project. This showed good technique had continued with increased awareness of the closed method for sampling as first choice method. There were a total of $817 \mathrm{BC}$ sampled with 16 contaminated $\mathrm{BC}$ collected giving a mean contamination rate of 2.0\% (Figure 2, Results Supplement). During this period there was a $15.1 \%$ true positive rate. This compares with the preceding 12 months where $672 \mathrm{BC}$ were requested with 31 contaminated samples, a mean contamination rate of $4.61 \%$ and a $14.0 \%$ true positive rate.

Initially the number of days between contaminated samples (Figure 1, Results Supplement) increased and the target was almost achieved between the first and second contaminated samples. Figure 2 (Results Supplement) shows the study SPC chart which highlighted special cause variation in the number of contaminated samples in February. This incident followed a changeover of ST. Three out of six of these had already worked in the department and thus did not repeat the induction programme. This meant they had not received the newly introduced $\mathrm{BC}$ information (PDSA 4). Another issue, raised at this time, was the need for assistance in obtaining BC from challenging patients. This prompted PDSA 5 which brought nurses in as stakeholders in order to provide support to the doctors taking samples. This saw a steady increase in the days between contaminated samples with the 30-day target being achieved between each of the last four contaminated samples. The longest period was 99 days between events (03 July and 10 October 2015) where 207 patients had samples taken. From May to November 2015 saw four consecutive successful contaminant free periods longer than the 30-day target.

The use of the BC sampling tray and venepuncture trolley received positive feedback. As there had been no modification suggestions, this format remained constant through the whole study period.

Case review of the contaminated samples showed that BCs were indicated for all cases and discussion highlighted a number of operator and patient factors that were present when contaminants were sampled. These represented a number of challenging cases that did not surprise the clinical team when informed of the contamination. Often they immediately identified patient factors which created difficulty or a clear deviation from the preferred technique. These reinforced the following common themes which were disseminated to the whole department (Appendix 2, Supplementary Material): follow the guidance for BC sampling paying close attention to the correct alcohol wipe and not re-palpating the vein prior to puncture; take your time and set up your workstation properly with the correct equipment intended for the procedure (preferably the closed system); and ask for help, e.g secure limbs with difficult patients. We also identified patient groups in whom a

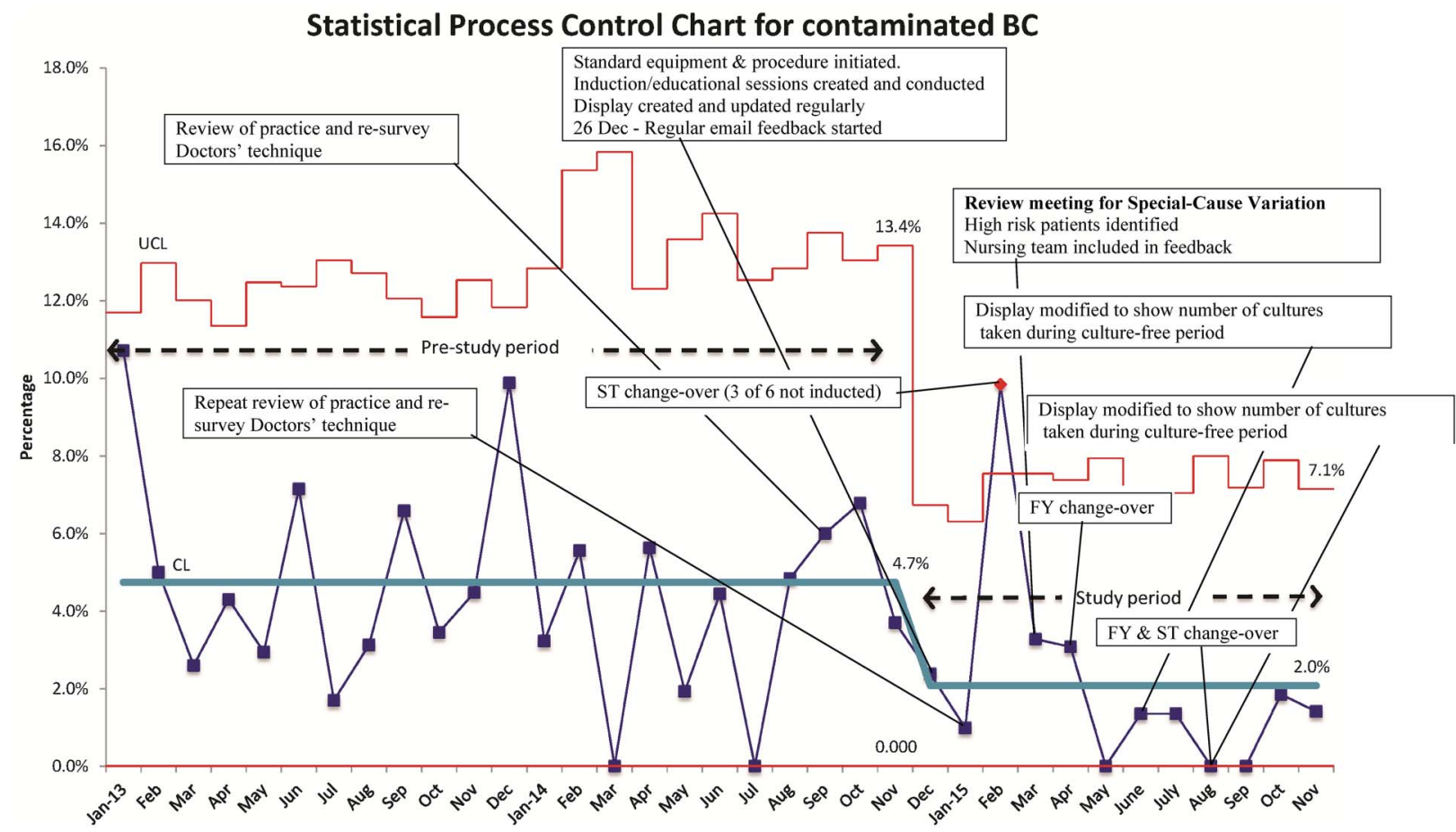

Figure 2 Statistical Process Chart (SPC) comparing monthly and mean contaminated BC rate for the pre-study period compared with the study period. 
greater number of BC contaminations occurred. These were: elderly patients ( $>65$ years old), which was the group who had the most BC taken and also more contaminations; and children ( $<15$ years old), for whom $\mathrm{BCs}$ were rarely required but when required the child was often in extremis and BC taken via a cannula. This information was frequently reiterated to staff through the email updates and displays (Appendix 2, Supplementary Material).

\section{LESSONS AND LIMITATIONS}

This study taught us a number of things:

Engaging stakeholders is imperative to instigate and maintain a successful change. Further to this it is important to engage those not directly responsible, but who may offer support. We had initially identified that, although not often required to take $\mathrm{BC}$, senior clinician buy-in was needed in order to encourage and support junior doctors using our preferred technique. We also learned that we needed to widen this enrolment to include nurses who have no direct responsibility or involvement in taking cultures but are involved in other aspects which could affect the ability to maintain the change.

We found that junior doctors were familiar with the safety devices available and favoured their use. Senior doctors favoured more traditional methods and may have previously steered junior colleagues to use their preferred method when supervised. Standardising technique and rationalising equipment eliminated any confusion as to which equipment or technique to use and allowed the practitioner to focus on performing the task. Our project suggests that good technique is sufficient to reduce $\mathrm{BC}$ contaminations and introducing sterile gloving techniques is not necessary. Easy access to the preferred method facilitated this. Senior doctors were more often involved in encounters with difficult patients who had failed or limited access in which using an alternative method would seem appropriate. When these led to contamination it was not unexpected by the clinical team and discussions could occur around what contributory aspects could have been improved or avoided.

By utilising the mandatory induction programme we were able to educate all the junior doctors rotating through the department. The benefit of this was supported by the increase in contaminated samples immediately after February when a number of returning doctors missed the education session. In future a small update session to the new department policies may be of use. Unfortunately, the drop-in sessions, were poorly attended and so the education to permanent staff occurred on an ad-hoc basis. Formal teaching settings might have achieved better outcomes, however these are difficult to achieve because of the 24-hour clinical demand on an ED. The regular emails with case feedback may have filled some of the void left through non-attendance to the drop-in sessions. These emails repeated common themes and learning points linking in with and directing stakeholders to the displays and may have stimulated some of the ad-hoc teaching sessions.

The learning points and common themes which came from our case review supported those suggested by other studies ${ }^{17-20}$. Our study was not designed to analyse these factors through statistical measures but to identify the important factors locally. The set-up of case discussion with those directly involved allowed these themes to come to light in a way that the clinical team could relate to their own practice and thus improve compliance with advice.

There was feedback that regularly disseminating information and positive results and the open discussion when investigating contaminated samples made stakeholders feel valued and kept them engaged in the project. Had we not been able to maintain enthusiasm and belief in our aims and methods then we would have been unlikely to have shown such improvement. A challenge will be maintaining this practice and enthusiasm when BC contamination surveillance decreases through the maintenance stage. There is a chance that the Hawthorn effect had an influence on results. To try to reduce this we will be continuing the information in the induction package with monthly display updates and observe whether this level of surveillance and reminder is adequate.

Displaying the educational material on the notice board was not the ideal method as we could not guarantee who would look at or read this. Updating the information daily did not always occur because of clinical work-load or staff leave. Also a positive culture could take up to seven days (48 hours to identify a positive organism and $\mathrm{BC}$ are incubated for five days before being declared negative) before it was confirmed as a contaminant. This meant that 37 days had to pass before you could confidently conclude the 30-day target had been achieved and the running total displayed was potentially inaccurate by this number of days. Possibly having updates once a week would reduce the chances of believing the target had been met when it truly had not.

We found that frequent changes to the display configuration were noticed and prompted people to read the concise display which reiterated education background and learning points. Discussion was stimulated with people approaching the QI teams with questions about BC contamination and confirming ways to reduce contamination. The regular positive email updates, again an imperfect form of information dissemination, prompted discussion and review of the display. Although no blame was shown people did not want to be responsible for a contaminated sample and made active attempts to reduce the risks.

\section{CONCLUSION}

BC contaminations were successfully reduced in our ED by clearly defining preferred technique with a simple 
and clear checklist and rationalising equipment to support and not distract from this method. This was supported by a realistic and simple, but competitive, target which would ensure an appropriate contamination rate along with regular positive updates on how well this target was achieved with advice on how to improve practice. Involving the larger clinical team in formulating this advice engaged members and promoted adherence to the factors needed to achieve the goals.

Ninewells ED is one of two EDs operated by NHS Tayside, the other being the ED of a District General Hospital, Perth Royal Infirmary, which operates with the same medical personnel and on-site senior doctor cover Monday to Friday 09.00-17.00. The interventions have already been rolled out to this site with positive results. This added an element of competition between the departments which increased the desire to reduce the number of contaminated samples. In Tayside admissions are referred directly to hospital specialties from the community without passing through the ED. This means a similar cohort of patients would be assessed on the Acute Medical Unit (AMU) and the Acute Surgical Receiving Unit (ASRU). The results have been fed back to the Microbiology Department who are supporting further dissemination to these two similar units within the organisation and will provide them with the same essential laboratory information and results to create up-to-date and targets and allow frequent staff updates. The challenge would be to engage the senior doctors and nurses on these units to support the interventions presented by this study. With evidence that these interventions can work on similar local units, which operate slightly differently to the ED, would support the benefit of other organisations adopting these methods to reduce $\mathrm{BC}$ contamination rates.

Acknowledgements All ED and Microbiology staff. Gabby Phillips (Consultant Microbiologist and Lead Infection Control Doctor, NHS Tayside) for assistance and guidance.

\section{Declaration of interests Nothing to declare.}

Ethical approval The work being reported was deemed exempt from ethics review. The work is primarily intended to improve local care, not provide generalisable knowledge in a field of inquiry.

Open Access This is an open-access article distributed under the terms of the Creative Commons Attribution Non-commercial License, which permits use, distribution, and reproduction in any medium, provided the original work is properly cited, the use is non commercial and is otherwise in compliance with the license. See:

- $\mathrm{http}: / /$ creativecommons.org/licenses/by-nc/2.0/

- http://creativecommons.org/licenses/by-nc/2.0/legalcode

\section{REFERENCES}

1. Gov.uk. [Internet]. Taking Blood Cultures A summary of Best Practice. [updated 2010 July 2010; cited 20117 Apr]. Department of Health (United Kingdom). Available from: http://hcai.dh.gov.uk/files/ 2011/03/Document_Blood_culture_FINAL_100826.pdf.

2. Dellinger RP, Levy MM, Carlet JM, et al. Surviving Sepsis Campaign: international guidelines for management of severe sepsis and septic shock: 2008. Crit Care Med 2008;36:296-327.
3. Murphy O, Freeman R. Bacteriology of normal sterile body fluids. In: Hawkey P, Lewis D, editors. Medical Bacteriology. Second Oxford: Oxford University Press; 2004. p. 27-53.

4. Timbury MC, McCartney AC, Thakker B, Ward K, N. Notes on Medical Microbiology. Edinburgh: Churchil Livingstone; 2003.

5. Archer GL. Coagulase-negative staphylococci in blood cultures: the clinician's dilemma. Infect Control 1985;6:477-78.

6. Thwaites GE, Edgeworth JD, Gkrania-Klotsas E, et al. Clinical management of Staphylococcus aureus bacteraemia. Lancet Infect Dis 2011;11:208-22.

7. Weinstein MP, Towns ML, Quartey SM, et al. The clinical significance of positive blood cultures in the 1990s: a prospective comprehensive evaluation of the microbiology, epidemiology, and outcome of bacteremia and fungemia in adults. Clin Infect Dis 1997;24:584-602.

8. Gov.uk. [Internet]. Antimicrobial resistance and healthcare associated infections (AMRHAl) reference unit is the national reference laboratory for investigating antibiotic resistance in healthcare associated bacterial pathogens. [updated 20141 July; cited 2014 Nov]. AMRHAI reference unit: reference and diagnostic services. Available from: https://www.gov.uk/guidance/ amrhai-reference-unit-reference-and-diagnostic-services.

9. Richter SS, Beekmann SE, Croco JL, et al. Minimizing the workup of blood culture contaminants: implementation and evaluation of a laboratory-based algorithm. J Clin Microbiol 2002;40:2437-2444.

10. Richter SS. Strategies for minimizing the impact of blood culture contaminants. Clin Microbiol Newsl 2002;24:49-53.

11. Rogers KL, Fey PD, Rupp ME. Coagulase-Negative Staphylococcal Infections. Infect Dis Clin North Am 2009;23:73-98.

12. Shafazand $\mathrm{S}$, Weinacker $\mathrm{AB}$. Blood cultures in the critical care unit: improving utilization and yield. Chest 2002;122:1727-36.

13. Seigel TA, Cocchi MN, Salciccioli J, et al. Inadequacy of Temperature and White Blood Cell Count in Predicting Bacteremia in Patients with Suspected Infection. J Emerg Med 2010.

14. Metersky ML, Ma A, Bratzler DW, et al. Predicting bacteremia in patients with community-acquired pneumonia. Am J Respir Crit Care Med 2004;169:342-7.

15. Alahmadi YM, Aldeyab MA, McElnay JC, et al. Clinical and economic impact of contaminated blood cultures within the hospital setting. $J$ Hosp Infect 2011;77:233-6.

16. Bates DW, Goldman L, Lee TH. Contaminant blood cultures and resource utilization. The true consequences of false-positive results. JAMA. 1991;265:365-369.

17. Hall KK, Lyman JA. Updated review of blood culture contamination. Clin Microbiol Rev 2006;19:788-802.

18. Weinstein MP. Blood culture contamination: persisting problems and partial progress. J Clin Microbiol 2003;41:2275-78.

19. Eskira S, Gilad J, Schlaeffer P, et al. Reduction of blood culture contamination rate by an educational intervention. Clin Microbiol Infect 2006;12:818-21.

20. Self WH, Speroff T, Grijalva CG, et al. Reducing blood culture contamination in the emergency department: an interrupted time series quality improvement study. Acad Emerg Med 2013;20:89-97.

21. Kim N, Kim M, Lee S, et al. Effect of Routine Sterile Gloving on Contamination Rates in Blood CultureA Cluster Randomized Trial. Ann Intern Med 2011;154:145-151.

22. Infection Control \& Management Committee. Sharps Injury Prevention Strategy for NHS Tayside Employees. Dundee: NHS Tayside; 2014.

23. McBryde ES, Tilse M, McCormack J. Comparison of contamination rates of catheter-drawn and peripheral blood cultures. J Hosp Infect 2005;60:118-121.

24. Stohl S, Benenson S, Sviri S, et al. Blood cultures at central line insertion in the intensive care unit: comparison with peripheral venipuncture. J Clin Microbiol 2011;49:2398-2403.

25. Kelly AM, Klim S. Taking blood cultures from a newly established intravenous catheter in the emergency department does not increase the rate of contaminated blood cultures. Emerg Med Australas 2013;25:435-8.

26. Benenson RS, Kepner AM, Pyle DN, 2nd, et al. Selective use of blood cultures in emergency department pneumonia patients. J Emerg Med 2007;33:1-8.

27. Kennedy M, Bates DW, Wright SB, et al. Do emergency department blood cultures change practice in patients with pneumonia? Ann Emerg Med 2005;46:393-400.

28. Daniels R, Nutbeam T, McNamara G, et al. The sepsis six and the severe sepsis resuscitation bundle: a prospective observational cohort study. Emerg Med J 2011;28:507-12. 